\title{
1979 MEMBERSHIP DIRECTORY
}

(Corrected as of February 1979)

\author{
Permssion for Commercial Use Must be Obtained From \\ the NALLD Executive Secretary
}

A/V LANGUAGE JOURNAL, Birmingham B4 7ET, England

ABEL, JEAN P., Rd. 2 Martin Hill Road, Corning, NY 14830

ABEYTA, JERRY, BOX 674, Center, CO 81125

ACADIA UNIVERSITY, Vaughn Memorial Library, Wolfville, NS BOP 1X0, Canada

ADAMS, MRS. SUDIE M., Baylor University, Spanish and Portuguese, Box 159, Waco, TX 76703

ADFL BULLETIN, Editor, 62 Fifth Avenue, New York, NY 10011

AIKENS, H. F., Director, Dalhousie University, Language Laboratory, Halifax, NS, Canada

ALLEN ,ERNEST, Director, Texas Christian University, Language Laboratory, 1 Allen Farm Road, Ft. Worth, TX 76116

ALLEN, TED, Laboratory Director, ELS Language Center, UD Station, Irving, TX 75061

ALLEY, GARY J., Language Lab Manager, Department of State, Foreign Service Institute, 1400 Key Blvd., Arlington, VA 22209

ALIANCE FRANCAISE EAST BAY, POB 11138, Oakland, CA 94611

ALTMAN, DR. HOWARD B., University of Louisville, Dept. Modern Language, Louisville, KY 40208

ALTMAN, LEON, Director, San Francisco State College, Foreign Language Laboratory, 1600 Holloway, San Francisco, CA 94132

AMANO, KAZUO, 16-6 Shiomigaoka-Cho, Chiba-SHI 280, Japan

AMER COUN TEACH FOR LANGS, Attn: David P. Benseler, Editor, For. Lang. Annals, Ohio State University, Dept. of German, Columbus, OH 43210

ANASA, VILLETTE, Adelphi University, Language Laboratory, Garden City, NY 11530

ANASTOS, PERRY, 155 E. 33rd Street, New York, NY 10016

ANDREWS, M. L., Language Lab Supervisor, University of Otago, Box 56, Dunedin, New Zealand

APPLACHIAN STATE, Periodicals Department, University Library, Boone, NC 28608

APPLIED LIGUISTICS, INST. OF WILLY MARTIN, Blijde Inkomststraat 21, 3000 Leuven, Belgium

ARIZONA STATE UNIVERSITY, Library Periodicals, Tempe, AZ 85281

Spring/Summer, 1979 
ASSELIN, JEAN-GUY, Supervisor, Carleton University, Language Laboratory, 126 Paterson Hall, Ottawa, ON KIS 5B6, Canada

ASSELT, IAN VAN, Head, McPherson College, Dept. Foreign Languages, McPherson, KS 67460

ASSOCIATION FOR EDUCATIONAL, Communication and Technology, John J. Faber, Advertising Manager, 1201 Sixteenth St. N.W., Washington, DC 20036

AUCOIN, G.E., 34 Langley Ave., Dartmouth, NS B2W 2Y6, Canada

AULESTIA, VICTOR H., Director, University of Maryland, Language Media Center, 5401 Wilkens Avenue, Baltimore, MD 21228

AUSTRALIA, NAT'L LIBRARY OF PRELIMINARY PROCESSING, (NS 110/72), Canberra, A.C.T. 2600, Australia

AUSTRALIAN NAT'L UNIV., Instructional Resources Unit, Chifley Library, Box 4, Canberra A.C.T. 2600, Australia

AVEDEX INC., 7326 Niles Center Road, Skokie, IL 60076

BABIN, J. W., Assoc. Prof., French, St. Francis Xavier Univ., Antigonish, NS B2C 1C0, Canada

BAILEY ,LESLIE F., Director, 48B Kresge, Language Laboratories, Northwestern University, 1859 Sheridan Road, Evanston, IL 60201

BAKER, REID E., Ohio Dept. of Education, 65 S. Front Street, Columbus, OH 43215

BALDWIN, VIRGINIA, Dept. Chairman, Crystal Lake High School, 45 W. Franklin St., Crystal Lake, IL 60014

BALL STATE UNIVERSITY, Continuations, Dept. of Library Service, Muncie, IN 47306

BALLEW, EMILY MAE, Instructor, Northwest Mississippi J.C., Modern + Foreign Languages, Senatobia, MS 38668

BARR, CHARLES J., Director, St. John's College, Language Laboratory, 5118 Seminary Road, Camarillo, CA 93010

BAUMAN, MR. RONALD A., Dept. of Mod. European Langs., Pomona College, Claremont, CA 91711

BECK, FRANCES J., University of Chicago, 5832 South Stoney Island, Chicago, IL 60637

BENNINGHTON COLLEGE, Director, Language Laboratory, Bennington, VT $0520 \mathrm{~L}$

BENT, ANTHONY J., 140 Brandeis Road, Newton, MA 02159

BERKELEY HIGH SCHOOL, C/O H Lorenzo, foreign Languages, Grove \& Bancroft, Berkeley, CA 94704

BERLIN, FREE UNIVERSITY OF FOREIGN LANGUACE LABORATORIES, Habelsch Werdter Allee 45, Ihnenstrasse 24, 1 Berlin 33 (Dahlem), Germany

BERNDT, DR. ROBERT J., Head, Southwest Missouri State Univ., Dept. of Foreign Language, Springfield, MO 65802

BERUBE, JEANETTE, SISTER, Director, Annhurst College, Language Laboratory, Woodstock, CT 06281

BETTELER, MICHAEL, Director of Courses, ELS Language Center, 5009 Calhoun Road, Houston, TX 77004 
BLAKE, LORRAINE J., Language Lab Director, Eastern Nazarene College, 23 East Elm St., Quincy, MA 02170

BOUDREAU, JACQUELINE, Supervisor, Wheaton College, Language Laboratory, Norton, MA 02766

BOUGARD, DR. ROGER, Lab Director, NCCU, Box 19371, Durham, NC 27705

BOWEN, LOUISE H., Dartmouth College, 201 Bartlett Hall, Hanover, NH 03755

BOWIE STATE COLLEGE, Thurgood Marshall Library, Periodical Dept., Bowie, MD 20715

BOYDEN, PARTICK C., Director, Kent State University, Language Laboratory, Kent, OH 44240

BRANNOCK JR., D. V., Assistant Professor, Clemson University, 201 Strode Tower, Clemson, SC 29631

BRAXTON JR, SHERROD L., Assistant Professor, French, Oral Roberts University, 7777 S. Lewis, Tulsa, OK 74171

BRESNICK, SOL, Coll. Lab. Technician, Queens College, 65-30 Kisseng Blud., Flushing, NY 11367

BRIDGERS, MR. W. K., Comm. Prod. Asst., University of California, Phelps Hall 1521, Santa Barbara, CA 93106

BRICCS, BECKY A., Michigan State University, Language Laboratories, A-126 Wells Hall, East Lansing, MI 48824

BRIGHAM YOUNG UNIVERSITY, Harold Lee Library, Serials Section, Provo, UT 84602

BRISTOL COMMUNITY COLLEGE, Library, 777 Eisbree Street, fall River, MA 02720

BRITISH LIBRARY, Accessions Department, Lending Division, Boston SPA, Wetherby, Yorkshire LS23 7BQ, England

BROSSMAN, DR. LUTHER F., Director, Albright College, Modern Language Laboratory, Reading, PA 19604

BROWN UNIVERSITY, Language Laboratory Library, Box E, Providence, RI 02912

BROWN, C. P., Western Kentucky University, Ivan Wilson Building 251, Foreign Language Department, Bowling Green, KY 42101

BRUNO, MARLINDA R., Director, Vanderbilt University, Language Laboratory, Box 6202, Station B, Nashville, TN 37235

BUCHHANDLUNG LEHMKUHL, 8000 Munchen 23, Leopoldstrasse 45, West Germany

BUNKER, DR. DUANE F., Assistant Professor, Dept. of Foreign Language, Appalachian State University, Boone, NC 28608

BURGGraAf, SAMUEL, E., Chairman, Dept. of Learning Res. Ctrs., Brigham Young University, 2330 Lee Library, Provo, UT 84602

BUTLER, MRS. HORTENSIA, Asst. Dean, Foothill College, Mountain View Center, 1965 San Ramon Ave., Mountain View, CA 94043

CALIF POLYTEChNIC STATE UNIV., University Library, Periodicals Section, SN LUIS OBSPO, CA 93407 
CALIF. STATE UNIV.-SACREMENTO, Spanish-Portuguese Dept., 6000 J Street, UPO \#479, Sacremento, CA 95819

CALIF-SAN DIEGo, UNIV. Of LANGUAGE LAB C-008, Muir College, La Jolla, CA 92093

CALLAHAM, DR., 820 North Michigan Avenue, Chicago, IL 60611

CALVIN COLLEGE LIBRARY, 3207 Burton S.E., Grand Rapids, MI 49506

CAPE BRETON, College of Sydney Campus, Library, Box 760, Sydney, NS B1P 611, Canada

CAPRETZ, PIERRE J., Director, Yale University, Language Laboratory, 111 Grove Street, New Haven, CT 06510

CARLETON COLLEGE, Library, Northfield, MN 55057

CARLSON, FLORENCE, Director, Wellesley College, Language Laboratory, Wellesley, MA 02181

CARTIER, JR., F. A., 1029 Forest Avenue, Pacific Grove, CA 93950

CATE, ROBERT L., Director, University of Texas-EI Paso, Modern Language Laboratory, EI Paso, TX 79968

CELANDINE, THOMAS P., Atlantic Community College, Mays Landing, N] 08330

CENTRAL STATES CONFERENCE, Dr. Maurice W. Conner, University of Nebraska, P.O. Box 688, Omaha, NE 68101

CHANG, GERALD K. J., University of Hawaii, Foreign Language Laboratory, Moore Hall 256, 1890 East-West Road, Honolulu, HI 96822

CHAPMAN, DAVID CRAIG, Learning Resources. University of California, Santa Barbara, CA 93106

CHEATHEM, ROSALIE M., Director, Univ. of Arkansas-Little Rock, Language Laboratory, 6405 Evergreen Road, Little Rock, AR 72207

CHERRY, CHARLES MAURICE, Director, Furman University, Modern Language Laboratory, Greenville, SC 29613

CHRISTMAN, HENRY R., Director, Kutztown State College, Language Laboratory, Kutztown, PA 19609

CICERAN, A. I., Supervisor, Brock University-Decew Campus, Language Laboratory, St. Catharines, ON L2S-3AI, Canada

CLAMEN, JOEL, 88 Horner Avenue, Toronto, ON M8Z 4X8, Canada

CLAMEN, JOEL, 88 Horner Avenue, Toronto, ON M8Z 4X8, Canada

CLAMEN, JOEL, 88 Horner Avenue, Toronto, ON M8Z 4X8, Canada

CLARK, MR., John Hopkins University, Room 11 Gilman Hall, Charles \& 34th Streets, Baltimore, MD 21218

CLEVELAND STATE UNIVERSITY, Library Order Unit-Serials, 1860 E. 22nd Street, Cleveland, $\mathrm{OH} \mathbf{4 4 1 1 5}$

COE, GAYLE, Audio-Visual Technician, Cleveland State University, 3487 W. 122 St., Cleveland, $\mathrm{OH} 44111$

COHAN, HELENE C., Director, Language Lab, Beaver College, Dpt. of Foreign Languages, Clenside, PA 19038 
COLE, BENNETT, Asst. Professor, Spanish, University of Delaware, Dept. of Langs. \& Lit., Newark, DE 19711

COLE, DR. BRIAN A., Coordinator, Anne Arundel Community College, Learning Center, 101 College Parkway, Arnold, MD 21012

COLORADO, UNIVERSITY OF LIBRARY, Serials, Boulder, CO 80309

COMET, HONORE, Director, All-World Language Institute, Suite 500, Language Laboratory, 280 South Beverly Drive, Beverly Hills, CA 90212

CONCEPCION, UNIVERSIDAD DE, Biblioteca Central, Casilla 1807, Concepcion, Chile

CONCORDIA UNIVERSITY, Sir George Williams Campus, Library, Serials Acquisitions, 1455 Maisonnouve Blvd. West, Montreal, Quebec H3G 1M8, Canada

CONWELL, DR. MARILYN J., 251 W. Dekalb Pike, KNK of Prusia, PA 19406

COOPER, C. T., Assistant Professor-Spanish, Lebanon Valley College, Annville, PA 17003

COUROUX, GERALD, College Marie-Victorin, 7000 Rue Marie Victorin, Montreal, PQ 462, Canada

COX, JERRY, Director of Language Lab, Furman University, Dept. of Modern Loreign Langs., Greenville, SC 29613

COX, SHIRLEY O., Director, Auburn University, Language Laboratory, Auburn, AL. 36830

CREAMER JR., JOHN F., Director, Language Lab, Hudson High School, Brigham St., Hudson, MA 01749

CRIMINALE, LEONARD R., Director, Elmira College, Language Laboratory, Elmira, NY 14901

CTR FOR INFO ON LANG TEACH, Deputy Librarian, 20 Carlton House, Terrace, London S.W.1Y 5AP, England

CUMMINCS, STEPHEN, 9 Fairfield Street, Cambridge, MA 02140

DAl, HIROSHIMA, Honbu Toshokan-K, Hiroshima $730 \mathrm{MZ}$, apan

DAIGAKU, GIFU JYOSHI TANKI, 2693 Fukumitsu, Nagara, Gifu 500 MZ, Japan

DAIGAKU, KYOTO SANKYO, Toshokan (Eigo-Ken), Motoyama Kamigamo, Kita-Ku, Kyoto $603 \mathrm{MZ}$, Japan

DAIGAKU, TOKYO-SEITOKU-TANK I, Library (MZ), 7-12, Jujodai-L-Chowe, Kita, Ku, Toyko, Japan

DARIEN HIGH SCHOOL, Foreign Language Dept., Darien, CT 06820

DAVILLA, ADRIAN, Director, ELS Language Center, Laboratory, 5009 Calhoun Road, Houston, TX 77004

DAY, STEPHEN, Assistant Professor-French, Queen's University, Kingston( ON, Canada DAYTON, UNIVERSITY OF LIBRARY, Periodicals Department, Dayton, OH 45469

DE LA SALLE COlLEGE, Library, P.O. Box 3819, 2401 Taft Avenue, Manila, Philippines DEBONE, DR. G., Head, Lagrange College, Language Department, Lagrange, GA 30240

DEANOEYE, ROSA E., Language Lab Director, California St. University-La, 2400 Lyric Avenue, Los Angeles, CA 90027

DELATTRE, MME E., Crapel-Cretaal, Univ. De Nancy II, B.P. 33.97, 54015 Nancy Cedex, France 
DELONG, EDWARD JAMES, 6634 Three Clopt Road, Richmond, VA 23226

DEPALMA, VIRGINIA QUINTANA, Laboratory Director, Universidad De EI Salvador, Department De Idiomas, San Salvador, El Salvador, Central America

DETI, DANIEL V., Chairman, Language Department, College of the Desert, Palm Desert, CA 92260

DETORRE, EMILIO E., Queens College, Cuny, Flushing, NY 11367

DEURtOO, MRS. W. C., University of Alberta, Romance Language Lab, 24 Arts Building, Edmonton, AB, Canada

DeVARGAS, DeloreS, Director, Instituto De Idiomas, Carilla 51, Tarija, Bolivia, South America

DICKERMAN, WILLIAM C., 16439 Brookford, Houston, TX 77058

DIO, Procurement Division, Attn: Mrs. Connors/XL, PO Box 325, Ft. Ord, CA 93941

DIO, Procurement Division, Attn: Mrs. Connors/XL, PO Box 325, Ft. Ord, CA 93941

DIO, Procurement Division, Attn: Mrs. Connors/XL, PO Box 325, Ft. Ord, CA 93941

DIVINCENZO, VITA J., Director, Villanova University, Language Laboratory, Spring \& Hilldale Road, Malvern Rd. 2, PA 19355

DLIWC ACADEMIC LIBRARY, Building 618, Pres/Mtry, CA 93940

DLIWC ACADEMIC LIBRARY, DAKF03-77-M-3705, Building 302, Pres/Mtry, CA 93943

DLIWC ACADEMIC LIBRARY, DAKF03-77-M-3705, Building 302, Pres/Mtry, CA 93943

DLIWC ACADEMIC LIBRARY, DAKF03-77-M-3705, Building 302, Pres/Mtry, CA 93943

DOCK, STEPHEN V., Coordinator, Vanderbuilt University, Vanderbuilt Language Lab, Box 1651 Station B, Nashville, TN 37235

DODD, KEN, West Georgia College, School of Arts \& Sciences, Dept. of Foreign Languages, Carollton, GA 30118

DOWLINC, DR. I. K., Director, University of Queensland, Language Laboratory, St. Lucia, Brisbane 4067, Australia

DROBNIC, KARL, Lab Instructor, ELI, Oregon St. University, PO Box 487, Waldport, OR 97394

DUKAS, VYTAS, San Diego State College, Language Laboratory, 5402 College Avenue, San Diego, CA 92182

DZELME, SILVIJA, Head, Broad Ripple High School, Foreign Language Dept. 7769 Landings Drive, Indianapolis, IN $\mathbf{4 6 2 4 0}$

EASTERN MONTANA COLLEGE, Library, Serials Department, Billings, MT 59101

EATON, SHIRLEY, 140 W. River St., Apt. E, Wilkes-Barre, PA 18702

ECONOMOS, R. M., Pace Universfity, 41 Park Row, New York, NY 10038

EDUCATIONAL ELECTRONICS OF CALIFORNIA, Bernard Keach, 213 North Cedar Avenue, Inglewood, CA 90301

EDUCATIONAL MEDIA DIVISION, Jim Goodin, P.O. Box 20604, 2812 Quail Plaza Drive, Oklahoma City, OK 73120 
EK, JAMES D., 16216 N. 33rd Drive, Phoenix, AZ 85023

EL PASO COMMUNITY COLLEGE, 6601 Dyer Street, El Paso, TX 79904

EL PASO PUBLIC SCHOOLS, West Area Office, C/O S. Sigueiros, 2231 Arizona Street, EI Paso, TX 79930

ELDRIDGE, MR. RICHARD H., Dept. of Humanities, Florida Memorial College, 15800 NW +2nd Ave., Miami, FL 33054

ELLIS, DONNA L., Language Lab Director, West Chester State Collge, Foreign Language Dept., West Chester, PA 19380

ELMENDORF, WILLIAM E., Director, Western Washington State Col., Language Laboratory, Bellingham, WA 98225

ENGLAND, DAVID, Communications Lab, North Lake College, Comm/Hum Division, 2000 Walnut Hill Lane, Irving, TX 75062

ENTIN, DR. NATHANIEL A., Gratz College, 10th Street and Tabor Road, Philadelphia, PA 19141

ERIC EXCHANGE, LR/, Center for Applied Linguistics, 1611 N. Kent Street, Arlington, VA 22209

FAULKNER, JAMES C., State University College, 17 Cdar Ridge Road, New Paltz, NY 12561

FECTEAU, LEO J., Chairman, Barrington High School, Foreign Language Department, Barrington, RI 02806

FELSHER, DR. WILLIAM, Chairman, University of Evansville, Foreign Languages Department, P.O. Box 329, Evansville, IN 47702

fiCarRA, A. V., Assistant Professor, Western Conn. St. College, 181 White Street, Danbury, CT 06810

FISH, WILLIAM M., ELS Language Center, 415 Commonwealth Ave., Simmons College, Boston, MA 02215

FISHER, RONALD D., Director, LaSalle College, Language Laboratory, 20th Street at Olney Avenue, Philadelphia, PA 19141

FITCHBURC HIGH SCHOOL, Altn: John C. Baimas, F.L. Curriculum Leader, 98 Academy Streel, Fitchburg, MA 01420

FIORIDA STATE UNIVERSITY, Serials Department, R. M. Strozier Library, Tallahassee. FL 32306

FORQUER, MARY BETH, 29 East Cocpp Streel, Bethlehem, PA 18017

FRADKIN, BERNARD, Evergreen Valley College, 3095 Yerba Buena Road, San Jose, CA 95121

FRANCIS. LARRY P., Assistant Director, University of Missouri, Intensive English Center, 229 Gentry, Columbia, MO 65201

FRECHETTE, ERNEST A., DR., Florida State University, foreign Language Education, Tallanassec, FL 32306

FRIEDMAN, FRANK M., Director, C. S. Moll Community College, Language l.aboratories, 1401 E. Court Street, Flint, MI 48503

FRITSCH, HANS I., P.O. Box 61, Whately, MA 01093 
FROST, ARTHUR F., Director, Princeton University, Lanuage Laboratory, 304 E. Pyne Building, Princeton, NJ 08540

FRY, DAVID F., Supervisor, Wayne State University, Learning Systems, 5448 Cass Avenue, Detroit, MI 48202

FUKUOKA UNIVERSITY, LL Room, Nanakuma, Fukuoka 814 (MZ), Japan

GARfINKel, ALAN, DR., Associate Professor, Purdue University, Dept. of Modern Lang. Educ., Lafayette, IN 47907

GAUDINO, V. A., Modern Language Center, 252 Bloor Street West, Toronto, ON 818, Canada

GENDREAU, N., Rhode Island College, Modern Language Department, 600 Mount Pleasant Avenue, Providence, RI 02908

GENNAULA, M. G., 5872 Kings School Road, Bethel Park, PA 15102

GEORGE WASHINGTON UNIVERSITY, Romance Languages \& Lit., Washington, DC 20052

GEORGIA COLLEGE, Foreign Languages Dept., Milledgeville, GA 31601

GEORGIA INSTITUTE OF TECH., Library, 225 North Avenue NW, Atlanta, GA 30332

GEORGIA, UNIVERSITY OF LIBRARIES, Sets Department, Athens, GA 30602

GERMAN QUARTERLY, Editor, William A. Little, University of Virginia, Germanic and Slavic Lang., Charlotte SVL, VA 22901

GILL, S. ROSENDA, Coordinator, Regis College, Language Laboratory, Weston, MA 02193

GIONET, ARTHUR J., DR., North Texas State University, Dept. For. Langs. \& Lit., Denton, TX 76203

GLEAVES, MR. GLEN, Language Laboratory, University of S. Alabama, Mobile, AL 36688

GOLDENBERG, MYRNA, English Department, Writing Center, Montgomery College, Rockville, MD 20850

GOLDSMITH, STANLEY, Director, Columbia University, Language Laboratory, 101-B Philosophy Hall, New York, NY 10027

GOLDSWORTHY, T. R., Director, Univ. of Wisconsin at Madison. Labs for Recorded Instruction, Van Hise Hall 279, 1220 Linden Drive, Madison, WI 53706

GOODWIN, AMIE M., MS., Supervisor Language Lab, Boston State College, Media \& Instruc. Development, 625 Huntington Ave., Boston, MA 02115

GORDON, DAVID L., Director, Marquette University, Dept. of Foreign Lang. \& Lit., Language Laboratories, 526 North Fourteenth Street, Milwaukee, WI 53233

GORE, PETER B., Director, Deerfield Academy, Language Laboratory, Deerfield, MA 01342

GOULET, NOELLA, SR., French Teacher, Notre Dame College, 2321 Elm Street, Manchester, NH 03104

GREAT FAllS, COLLEGe OF LIBRARY, Periodicals Dept., 1301 20th Street South, Great Falls, MT 59405

GREEN, RUTH L., Director, Williams College, Language Laboratory, Karl E. Weston Lang. Center, Williamstown, MA 01267 
GRIGC, BILLIE, MRS., Supervisor, El Camino College Lang. Lab, Torrence, CA 90506

GROSJEAN, GLEN, Assistnt Director, University of California, Language Laboratory, B-40 Dwinelle Hall, Berkeley, CA 94720

GROSS, STEPHANIA E., Bowling Green State Univ., University Hall, Bowling Green, $\mathrm{OH} 43402$

GUITTON, JEAN, DR., Chairman, Georgia College, Modern Language Laboratory, Milledgeville, GA 31061

HALL, BUNELLE M., A-V Corrdinator, Monterey Institute for Foreign Studies, 440 Van Buren Street, Monterey, CA 93940

HAMM, PROFESSOR C., Director, Queen's University, Language Laboratories, Kingston, K7L 3N6, Canada A7778

HAMMERLY, HECTOR, Simon Fraser University, Applied Linguistics, Department of Modern Langs., Burnaby, BC V5A IS6, Canada

HAMSON, ERWIN M., Director \& Lecturer, University of Michigan, Language Laboratory, 2003 Modern Lang. Building, Ann Arbor, Ml 48104

HANDELSMAN, MICHAEL, Asst. Prof., University of Tennessee, Dept. of Romance Languages, 601 McClung Tower, Knoxville, TN 39716

HANSON, DAVID A., Assistant Professor, Brandeis University, Listening Center, Waltham, MA 02154

HANZEL SVD, REV. LOUIS F., Nanzan University, 18 Uamatzato-Cho, Showa-Ku, Nagoya 466, Japan

HANZELI, PROFESSOR VICTOR, Director, Language Learning Center, 116 Denny Hall DH-40, University of Washington, Seattle, WA 98195

HARJAN, PAULA, Lang. Lab. Supervisor, York University, Ross Bldg. 5115, 4700 Keele Street, Downsview, ON M3] IP3 , Canada

HARRISON, JOHN S., Foreign Languages, Board of Education of Baltimore County, Towson, MD 21204

HARTLEY, G. CHRISTOPHER, Head, The Peddie School, Language Department, Hightstown, NJ 08520

HAWKINS, CORNELIA, Librarian, Ross Memorial Library, Cottey College, Ross Memorial Library, Nevada, MO 64772

HEALY, ANNE, Supervisor Language Lab, Boston College, Lyons 313, Chestnut Hill, MA 02167

HEATON, J., A-V Specialist-Lecturer, TESL Department, UCLA, Los Angeles, CA 90024

HENNIG, C. W., University of Waterloo, Language Laboratory, Room 110 ML Building, Waterloo, ON, Canada

HENRY, MARTIN A., MR., Director, Language Lab, Box 172, Gardiner, NY 12525

HERNANDEZ, ADELE B., Instructor, University of Maine, Department of Spanish, 96 Portland, ME 04103

HERNONDEZ, IRIS, Professor, Pensacola Junior College, Language Laboratory, Pensacola, FL $325 \$ 3$

HERRERA, LAZADO M., Director, The Westminister Schools, Language Laboratory, 1424 W. Paces Ferry Road N.W., Atlanta, GA 30327 
HEWSON, J., DR., Head, Memorial U. of Newfoundland, Department of Linguistics, St. John's, NF, Canada

HILTS, MRS. MARGARETE, Loma Linda University, La Sierra College, Riverside, CA 92505

HIRSCH, B. G., Director, Samford University, Language Laboratory, 800 Lakeshore Drive, Birmingham, AL 35209

HISPANA, Advertising Manager, Alfred Turner, Glenbrook South High School, 4000 West Lake Avenue, Glenview, IL. 60025

HISPANIA, Editor, Donald W. Bleznick, University of Cincinnati, Romance Languages, Cincinnati, $\mathrm{OH} 45221$

HOARD, Foreign Language Laboratory, University of Oregon, 14 Friendly Hall, Eugene, OR 97403

HOELDERLE, MS., Woodrow Wilson High School, 31st \& Federal Streets, Camden, NJ 80101

HOLMES, GLYN, DR., Director of Lang. Labs/French, Department of French, Univ. of Western Ontario, London, ON N6A3K7, Canada

HOLTON-ARMS SCHOOL, Library, 7303 River Road, Bethesda, MD 20034

HONG, SUNG MYUN, Director, Instructional Media Ctr, LTC, Kyung Hee University, SEOUL 131, Korea

HOWARD UNIVERSITY, Language Lab/903301, Room 361 Locke Hall, 2441 6th Street NW, Washington, DC 20059

HOWELL, JOEL T., Director, North Texas State University, Language Laboratories, Department of Foreign Langs., Denton, TX 76201

HOWLING, ROBERT T., Professor, New Have nCollege, English Department, New Haven, CT 06505

HUGHETT JR., HARVEY L., Supervisor, Language Lab, Department of Foreign Langs., University of Idaho, Moscow, ID 83843

HUTTEN, JEAN R. R.N., Coordinator of Learning Lab, Indiana University, School of Nursing, $1100 \mathrm{~W}$. Michigan, Indianapolis, IN 46202

I/C Library, Research Officer, Cent. Inst. of Indian Langs., Bharatiya Bhasha Sansthan, Manasagangsti, Myore-570 $006 \mathrm{~S}$. India

IANNUZZI, D. A., Chairman, Niagara University, Modern Languages, Niagara, NY 15109 INST FUR DOLMETSCHER AUS, Building, University Innsbruck, Innsbruck 6020, Austria INSTITUT DE LINGUISTIQUE, Appliquee, Laboratories De Langues, University De Strasbourg, 22 Rue DeScartes, 67084 Strasbourg-Cedex, France

INSTRUCTIONAL MATERIALS CTR, 201 East Nine Mile Road, Highland Spgs, VA 23075

INTERNATIONAL FILM BUREAU, Attn: Ms. Anne Herbert, 332 South Michigan Avenue, Chicago, IL 60604

IRVING, EVELYN, DR., 1840 Azrock Drive, Knoxville, TN 37914

IZUMI, MASUKO, Director Language Lab, Seinan Gakuin University, 6-2-92 Nishijin Nishi-Ku, Kukuoka 814, Japan

JACKSON, GORDON, Director, Capital University, Language Laboratory, 2199 E. Main Street, Columbus, OH 43209 
IARLETT, FRANCIS G., Language Laboratory Uilectur, Central Connecticut State Col., Modern Language Department, New Britain, CT 06050

JEWISH NAT'L \& UNIV. LIBRARY, Periodicals Department, P.O. Box 503, Jerusalem, Israel

JOCUMS, GEORGE, DR., Chairman, Foreign Language Dept., Boise State University 1910 University Drive, Boise, ID 83725

JOHNS, ALAN, Electronic Tech, Windsor University, French Lang \& Lit Department, Sunset Avenue, Windsor II, ON, Canada

JOHNSON, BARBARA, Washington State University, Foreign Language Department, Pullman, WA 99163

JOHNSON, MARY KATHERINE, 1720 Coventry Road, Cleveland Hts., OH 44118

JORDAN, T. C., Director, Griffith University, Language Centre, Nathan Queensland 4111, Australia

KACAWA UNIVERSITY, Library, Saiwai-Cho, Takamatsu-Shi, 760 (Kin), Japan

KAMEHAMENA SCHOOLS, Attn: Bill Gemmer, Comm. Arts/Language Dept., Bishop Hall, Kapalama Heights, Honolulu, HI 96817

KANSAI UNIVERSITY, Library, P.O. Box 50 Suita, Osaka 564 MZ, Japan

KATH, RUTH ROBERT, Route \#1, Box 187, Riverside, IA 52327

KATHOLIEKE UNIV. LeUVEN, Faculty of Letters \& Phil., Dept. of Linguistics, Section of Applied Ling., Blijde Inkomststraat 21, 3000 Leuven Belgium, Belgium

KAUPPAKOREAKDULU, Runeberginkatu, 14-16, Helsinki 10, Findland

KENNEDY, DORA F., Supervisor, Prince Georges's Public School, Foreign Language Department 4806 Harvard Road, College Park, MD 20740

KENTUCKY, UNIVERSITY OF, Language Laboratory, 312 Classroom Building, Lexington, KY 40506

KERR, CHARLOTTE W., Supervisor, Occidental College, Language Laboratory, 1600 Campus Road, Los Angeles, CA 90041

KESSLER, MICHAEL, Director, University of Houston, Foreign Languages Lab, Humanities \& Fine Arts, Houston, TX 77004

KHOURY, DWIGHT D., Director of Language Lab, Ohio Northern University, University Audio Center, ADA, OH 45810

KING, CAIL S., Director, Geddes Language Center, Boston University, 685 Commonwealth-Room 540, Bostun, MA 02215

KIRIASTO, JOENSUUN KORKEAK, PL 111, 8!)101 ICIENSUU 10, Findland

KIRK, DAVID, 124 Chase Strect, Santa Cruz, CA 95060

KITAO, KENII, Japan International Center, 39-1 Koyana-Shimofusa-Cho, Kita-Kuu, Kyoto, Japan

KOENEN, SHIRLEY, 2420 Appleside Blvd., Clarkston, WA 99403

KONRAD, KARL-Ludwig J., Direclor, astEern Illinuis University, Language Laboratory. Charleston, IL 61920

KONYVTAR, ECYETEMI, POB 483, Budgary 1372, Hungary 
KOPPEL, HELEN A., Director, Audio Laboratory/M73716, Tufts University, Eaton HallRoom 122, Medford, MA 02155

KOZORIZ, G., COMMANDANT, Canadian Forces FLS, Dept. of National Defense, Ottowa, ON 4, Canada

KRUSE JR., E. L., 733 Kirkwood Drive, Grand Island, NY 14072

KUMP, RICHARD J., 1822 Ricardo Drive, CPE Girardeau, MO 63701

KURITA, KATSUMI, Sony Language Laboratory, 6-5-8 Kitashinagawa, Shinagawa-Ku, Tokoyo 141, Japan

¿URIYAMA, SHOICHI, Language Laboratory, Waseda University, 1-6-1 Nishi-Waseda, Tokyo 160, Japan

ACEFIELD, ARCH S., Senior Instructor, University of Kentucky, Henderson Community College, College Library/Dav 798100, Henderson, KY 42420

ALLY JR., DALE V., Director, University of Louisville, Arts \& Sciences Learning Lab, Hum-Room 16, Louisville, KY $402044 \quad 444444$

AMERAND, R., DR., 252 Bloor Street West, Toronto, ON M5SLV6, Canada

LAMORE, DR. MARIE B., Director, Cottey College, Language Laboratory, Nevada, MO 64772

LANG \&LANG BeHAVIOR ABSTRACTS, P.O. Box 22206, San Diego, CA 92122

LANGE, DALE L., Professor, University of Minnesota, 148A Peik Hall, Minneapolis, MN 55455

LAWRASON, ROBIN E., DR., Director, Temple University, Media Learning Center, Philadelphia, PA 19122

LEIDECKER, CATHERINE, Assistant Principal, Americanization School, 3331 Prospect St. NW, Washington, DC 20007

LEMYZE, J. C., MCGill University, Language Laboratory, Peterson Hall Room 2, 3460 Mctavish, Montreal, PQ H3A IXC, Canada

LEONE, ARTHUR A., 116 San Salvador Drive, Port Richey, FL 33568

LETHBRIDGE, UNIVERSITY OF, Library, Serials Section, 4401 University Drive, Lethbridge, Alta TIK $3 \mathrm{M} 4$, Canada

LEVINSON, MRS. BERNICE, Director Language Laboratory, Brooklyn College, Cuny, Dept. of Modern Langs \& Lits, Brooklyn, NY 11210

LIBRARY OF CONGRESS, Ord. Division, Altn: Section C, Washington, DC 20540

LIDII, MARCEL, Lecturer, City College of New York, Romance Language Laboratory, 133rd \& Convent Avenue, New York, NY 10031

LINGUAPHONE INSTITUTE LTD., 207/209 Regent Strect, London, England

LOEW, RICHARD A., Director, Language \& Learning Hall, 126 Clemens Hall, Amherst, NY 14260

LONC BEACH CITY COLLEGE, Alin: Lorraine Yocky, Foreign Language Department, 4901 East Carson Street, Long Beach, CA 90808

LOOK, R. KENT, Classroom \& Tech Staff Supr., U. of California-Berkeley, Language Laboratory, B-40 Dwinelle Hall, Berkeley, CA 94720

LORE, A. C., DR., Dirertor, University of North Carolina, Language Laboratory, 105 Dry Hall, Chapel Hill, NC 27614 
LOUISVILLE, UNIVERSITY OF, Belknap Campus, Attn: Mrs. Spradlin, Library, Serials Department, Louisville, KY 40208

LOYD, JEANNE, Instructional Assistant, Community College of Denver, North Campus, 1001 E. 62nd Avenue, Denver, CO 80216

LUCKAU, PAUL F., Brigham Young University, Germanir Languages, 270 MSRD, Provo, UT 84602

LUCKER, M. S., Language Lab, Bldg. No. 14N-0641, Mass. Inst. of Technology, Cambridge, MA 02139

MA, RICHARD W. C., Learning Asst. Cntr. Dir., Hawaii Pacific College, 841 Bishop Street, Honolulu, $\mathrm{HI} 96813$

MACDONALD, MARY L., Assistant Manager, University of Guelph, Media Services, Arts Building, Room 017, Guelph, ONT NIH 2B5, Canada

MACINKO, JOHN, Director, University of Colorado, Language Labs, 302 Old Main Building, Boulder, CO 80302

MACKEY, CHARLES R., Simmons College/Foreign Langs., 300 The Fenway, Boston, MA 02115

MACKEY, LOLA A., 44 W. Thunderbird, Phoenix, AZ 85023

MAGILL, ROBERT, Director, Language Lab, Towson State University, Dept. of Modern Language, Baltimore, MD 21204

MAGIN, J. F., University of Western Ontario, London, ON 72, Canada

MANN SR., GARY D., Director, Ohio State University, Listening Center, Dieter Cunz Hall 155, 1841 Millikin Road, Columbus, OH 43210

MARESCHAL, ROGER E., 257 Avenue De La Colline, Aylmer, PQ 59J 1M2, Canada

MARIE CSFN, SISTER CECILIA, Nazareth Academy, Grant \& Torresdale Avenues, Philadelphia, PA 19114

MARIETTA COLLEGE, Modern Language Department, Marietta, OH 45760

MARINO, ANNA, Director, Hunter College, Language Laboratory, 695 Park Avenue, Box 375, New York, NY 10021

MARQUETTE UNIVERSITY, Dept. of Foreign Languages, 526 North 14th Street, Milwaukee, WI 53223

MARTIN, WILLARD, Director, Listening-Learning Service, The Pennsylvania State Univ., 13 Pinchot Mall, University Park, PA 16802

MARXHEIMER, EDWARD, Assoc. Professor, University of Alberta, Dept. of Romance Langs., Edmonton, AB T6C 2E1, Canada

MARYLAND, UNIVERSITY OF, Baltimore College, Library-Serials, 5401 Wilkens Avenue, Baltimore, MD 21228

MASON, RICHARD G., Lab Director, Michigan Tech. University, Humanities Department, Houghton, MI 49931

MASS./BOSTON, UNIV. OF, Harbor Campus, Attn: Media Center (Huttner), Dorchester, MA 02125

MCANDREW, ALEX, Director of Language, Study Center, University of Sydney, New South Wales, Sydney, 2006, Australia

MCCLURG, AVERY, Dept. of Lit. \& Lang., Eas\& Texas State University, Commerce, TX 75428 
MCCUNE, JERRY, Director, Indiana University, Language Laboratory, Ballantine Hall 120, Bloomington, IN 47401

MCGILL UNIVERSITY LIBRARIES, Serials Department, 3459 Mctavish Street, Montreal, PQ 112, Canada

MCNAB, JAMES P., Head, Virginia Polytechnic Inst., And State University, Foreign Langs. \& Lits. Dept., Blachsburg, VA 24061

MEDGAR EVERS COLLECE, City University of New York, 1127 Carroll Street, Brooklyn, NY 11225

MEIJI GAKUNI UNIVERSITY, Gaigo-Ken, 2-37, Shiroganedai L-Chome, Minatoku, Tokyo 108 (550), Japan

MELBORNE, UNIVERSITY OF, Language Centre Arts S, Parksville 3052, Victoria, Australia

MEMPHIS STATE UNIVERSITY, Department of Foreign Lang., Memphis, TN 38152

MENIN, J. L., Acting Director, Rice University, Language Laboratory, Rayzor Hall 106, Houston, TX 77005

MESSMER, LIDIA, Director, Univ. of Pennsylvania, Fas Audio Visual Center, Logan Hall, 249 S. 36th, Philadelphia, PA 19104

MESSNER, JR., C. A., Carleton College, Assoc. Prof. Mod. Lang.; Northfield, MN 55057 METCALFE, JOHN, 29 Willow Rd., Menlo Park, CA 94025

MEYER, MAMIE P., Director, Hamilton College, Language Laboratory, Clinton, NY 13323 MICKO, MICHALE, Director, Language Lab, Widener College, Chester, PA 19013 MILDENBERGER, ANDREA, 126 Asharoken Avenue, Northport, NY 11768

MILLER, J. B., Assistant Professor, Wittenberg University, Springfield, OH 45501

MILLER, SHELBY C., 31 Priest Road, Watertown, MA 02172

MILLER, WILLIAM I., DR., Asst. Prof. Modern Lang., University of Akron, 310 Olin Hall, Akron, OH 44325

MINISTRY OF CULTURE \& Recreation-Resource Centre, 77 Bloor St. West, 9th Floor, Toronto, ON M7A2R9, Canada

MINISTRY OF CULTURE \& RECREATION-CITIZENSHIP, Language Classes, 8 York Street, Toronto, ON M7A2R9, Canada

MINNESOTA, UNIVERSITY OF, Serial Records, Minneapolis, MN 55455

MISSOURI WESTERN STATE COLL., Attn: Sparks, 4525 Downs Drive, St. Joseph, MO 64507

MISSOURI, UNIVERSITY OF, At Columbia, Ellis Library, Acquisitions Department, Columbia, MO 65201

MODERN LAND. JOURNAL, Editor, Robert F. Roeming, University of Wisconsin, Milwaukee, WI 53201

MODERN LANG. JOURNAL, Business Manager, Wallace G. Klein, 13149 Cannes Drive, St. Louis, MO 63141

MODERN LANGUAGE ASSOCIATION, Advertising Manager, 62 fifth Ave., New York, NY 10011

MOLDIN, COLUMBUS M., Central Area Admin. Center, 6501 Lowland Drive, Landover, MD 20786 
MONASH UNIVERSITY, Periodicals Department, Clayton VIX 3168, Australia

MONGILLO, MRS. FRANCES, Assistant Director, Wesleyan University, Language Laboratory, Middletown, CT 06457

MOORE, J. MICHAEL, DR., Mesa College, San Diego, CA 92111

MOORHEAD STATE UNIVERSITY, Library-Serials Dept., Moorhead, MN 56560

MORRISON, ROBERT R., Southern Missionary College, P.O. Box 475, Collegedale, TN 37315

MOSER, WORTH S., Darlington School, Rome, GA 30161

MOSES, JAMES O., Assistant Professor, Stephen F. Austin St. Univ., Box 3042, Nacogdoches, TX 75962

MOUNTAIN, CLIFF, Director, Foreign Languages, Mesa College, Grnd Junction, CO 81501

MULHOLLAND, CHARLES V., Supervisor, Lang. Lab., University of Rhode Island, Department of Languages, Kingston, RI 02881

MULLA, MR. MOHAMED, 2385-6 Bishop, Ann Arbor, MI 48105

MURPHY, HAROLD T., Asst. Prof., Marshall University, Box 238, 16th and Third Street, Huntington, $W V 25701$

NANYANG UNIVERSITY, Library/Period Sect., Upper Jurong Road, Singapore, 22, Repuglic of Singapore

NARVAEZ, LEON, Assistant Professor, Romance Languages, Saint Olaf College, Northfield, MN 55057

NASH, DONALD S., Roberts Wesleyan College, North Chili, NY 14514

NASSAU COMMUNITY COllege, Library, Periodicals Unit, Attn: Ms. Magda Cantil, Stewart Avenue, Garden City, NY 11530

NATIONAL LIBRARY OF CANADA, Order Section Per 221424, 395 Wellington St., Ottawa, Ontario KIA ON4, Canada

NAVES, CARROLL E., Language Lab Director, Barry College, Miami, FL 33161

NeBRASKA, UNIVERSITY OF, Periodical Department, Gene Eppley Library, Box 688 Downtown Station, Omaha, NE 68101

NEUFChATEL, UNIVERSITE DE, Chaire De Linguistique Gen., Neufchatel, 2000, Suisse NEVADA-RENO, UNIVERSITY OF, Library, Serials Department, Reno, NV 89507

NEVADA, UNIVERSITY OF, Library-Periodicals Dept., Las Vegas, NV 89109

NEW MEXICO SCHOOL PROD. CO., P.O. BOX 2126, Albuquerque, NM 87103

NEW YORK STATE UNIVERSITY, Language Lab Director, 3435 Main Street, 7 Hayes C, Buffalo, NY 14214

NEW YORK STATE UNIVERSITY, E. H. Butler Library, 1300 Elmwood Avenue, Buffalo, NY 14222

NEY YORK, STATE UNIVERSITY OF Library, Serial Section, Vestal Parkway East, Binghamton, NY 13901

NEWCASTLE, UNIVERSITY OF, Serials Librarian, New South Wales 2308, Australia

NIEMAN, LINDA L., 11 Gary Place, Wappingry Fls, NY 12590 
NOBLE, DAVID, DR., Assoc. Prof. German Dept., Armstrong State College, Dept. of Langs. \& Literature, Savảnnah, GA 31406

NOJD, PROF. OLAVI, Director, Jyvaskyla University, Language Laboratory, SF-40 100 Jyuaskyla 10, Finland

NORRISH, N., Director Lang. Lab., Victoria University of Wellington Private Bag, New Zealand

NORTH HENNEPIN, Community College, Humanities Department, 7411 85th Avenue $\mathrm{N}$, Minneapolis, MN 55445

NORTHEAST CONFERENCE, James W. Dodge, Secretary Treasure, Box 623, Middlebury, VT 05753

NORTHROP WORLDWIDE AIRCRAFT, Service Inc., Attn: Librarian, Building 6237, Lackland AFB, TX 78236

NUFFER, J. STANLEY, Director, Learning Laboratory, Portland State University, P.O. Box 751, Portland, OR 97207

O'BRIEN, P. J., 280 Richmon dAve., Amityville, NY 11701

O'HEARON, SR. MARIE THERESE, Director of Language Lab., College of White Plains, 78 North Broadway, White Plains, NY 10603

OBERST, DR. B. S., Chairman, Dept. of Moedrn Languages, Cleveland State University, Cleveland, $\mathrm{OH} \mathbf{4 4 1 1 5}$

ODENSE UNIVERSITY, Language Laboratory, TSS Niels Bohr, Alle 75, DK-5000 Odense, Denmark

ODOM, WILLIAM, Department of Foreign Lang., Univ. of Southern Mississippi, Hattiesburg, MS 39401

OHIO STATE UNIVERSITY, Serials Division, 1858 Neil Avenue, Columbus, OH 43210

OKLAHOMA BAPTIST UNIVERSITY, Director Language Lab., Shawnee, OK 74801

OKLAHOMA STATE UNIVERSITY, Edmon Low Library, Serials Section, Stillwater, OK 74074

OLSEN, IRVING S., Director Valparaiso University, Instructional Materials, Valparaiso, IN 46383

ONTARIO INSTITUTE FOR STUDIES IN EDUCATION, Modern Language Center, LibraryPeriodicals, 252 Bloor Street W., Toronto, ON M5S IV6, Canada

QUELLETTE, R. J., Director, Language Lab, Southeastern Massachusetts, University, Old Westport Road, Group I Bldg. Rm 207, N. Dartmouth, MA 02747

P/H ELECTRONICS, 117 East Helena Street, Dayton, OH 45404

P/H ELECTRONICS, 117 East Helena Street, Dayton, OH 45404

PARK DLL, WILLIAM M., DR., Foreign Language Dept., University of North Carolina, Charlotte, NC 28213

PATRON, GLORIA A., Univ. of New Orleans, Language Laboratory, Lake Front, New Orleans, LA 70122

PAUCHET, JACQUELINE, Director Language Lab., Knoxville College, Knoxville, TN 37914

PAUL, MRS. C. E., Philadelphia College of, Pharmacy and Science, 43rd St. \& Gingsessing Ave., Philadelphia, PA 19104

PENNSYLVANIA STATE LIBRARY, Serial Records Section, Box 1601, Room 46, Harrisburg, PA 17126 
PENSACOLA JR. COLLECE, Library, 1000 College Blvd., Pennsacola, FL 32504

PENTA, RICHARD M., Coordinator of Foreign Lang., Belmont School System, 38 Normandy Road, Lexington, MA 02173

PEREZ, MARIO P., Director Language Lab., Brevard College, Brevard, NC 28712

PETERSEN, GERALD W., DR., Dept. of Foreign Languages, University of Nevada, Reno, NV 89507

PETHERBRIDGE, D. L., Assoc. Professor, University of Lethbridge, Lethbridge, AB, Canada

PFLANZ, BARBARA, Associate Professor, University of Redlands, Languages \& Literatures, Redlands, CA 92373

PIERCE, JAMES A., Instructor, Del Mar College, Department of English, Corpus Christi, TX 78404

PLEASANTS, PROF. J. V., Columbia University, 20 Dewey Lane, Hampton Bays, NY 11946

POLLY, LYLE R., Associate Professor, So. Missouri State Univ., Dept. of Foreign Languages, Springfield, MO 65802

PORT ELIZABetH, UNIVERSITY OF, Library-Periodicals Dept., Private Bag 6058, Port Elizabeth 6000 , South Africa

POTTAK, JUDY, Language Lab Technician, Bridgewater State College, Bridgewater, MA 02324

PROKOP, DR. MANFRED, Director, Language Labs, University of Alberta, Arts 203, Edmonton 7, AB T6G 2E6, Canada

PUERTO RICO, INTER-AMERICAN, University, Library, San German, PR 00753

PURDUE UNIVERSITY, Serials Unit-Library, W. Lafayette, IN 47907

QUEENS UNIVERSITY, Paul Klapper Librarq, Serials Section, Flushing, NY 11367

QUEENSLAND, UNIVERSITY OF, Serials Librarian, Main Library, St. Lucia, Queensland 4067, Australia

QUINN, T. J., Language Centre, Univ. of Melbourne, Parkville, Vic. 3052, Australia

RANDOLPH HICH SCHOOL, Foreign Language Coordinator, Memorial Parkway, Randolph, MA 02368

REESE, L. GRANT, Language \& Philosophy, UMC \#07, P.O. \#35732, Utah State University, Logan, UT 84322

REESE, WINSTON J., Language Lab Director, University of lowa, 125 Schaeffer Hall, lowa City, IA 52240

REEVES, GAYNOR E., Director, Univ. of Newcastle 2308, Language Laboratory, Newcastle, New South Wales, Australia

REEVES, PROF. DONA, Modern Language Dept., Southwest Texas State Univ., Rt. 2, Box 239A, Buda, TX 78610

REICH, SHELDON, 14-14 Wheatley Street, Far Rockaway, NY 11691

RENEGAR, CHRISTOPHER, Occidental College, Director, Language Lab, 1600 Campus Road, Los Angeles, CA 90041

RHODESIA, UNIVERSITY OF, Language Laboratory \#3643, P.O. Box MP 167, Mount Pleasant, Salisbury, Rhodesia 
RICE, THOMAS, 4000 Haverford Avenue, Riverside, CA 92507

RICHARDSON, MARTHA R., Assistant Director, Harvard University, Boylston Hall G-3, Cambridge, MA 02138

RICHARDSON, CHARLES P., Director of Language Laboratories, Ohio University, Athens, Ohio 45701

RICHMOND, EDMUN B., DR., Assistant Professor of German, Georgia Inst. of Technology, Atlanta, GA 30332

RICHTER, JOCHEN, Director of Language Lab, Allegheny College, Meadville, PA 16335

RICHTERICH, RENE, Director, Abteilung Fur Angewandte, Linguistik, Hallerstrasse 12, 3012 Berne, Switzerland

RIDDLE, BENTON E., Language Lab Director, Highland Park College, Highland Park, MI 48203

RIJKSUNIVERSITEIT TE, Groningen, Bibliotheek, Oude Kijk, In 'T Jatstraat 5, Postbus 559 (Netherlands), Netherlands

RIVERS, PROF. WILGA M., Department of Romance Lang., Harvard University, 207 Boylston Hall, Cambridge, MA 02138

ROBINSON, RICKEY, Supervisor, Media Distr. Sys., Governors State University, S. Pk Forest, IL 60466

RODEWALD, JANET D., Director of Language Lab, Central Michigan University, Mt. Pleasant, MI 48858

ROEDERER, JEAN LOUIS, Professor, Houghton College, Division of Foreign Langs., Houghton, NY 14744

ROGERS, J. J., DR., Director of Modern Languages, Lincoln Universtiy, Lincoln, PA 19352

ROWE, HAROLD M., Language Centre, Monash University, Clayton, Victoria 3168, Australia

ROWE, PROFESSOR GALEN O., Dept. of Foreign Langs \& Lit., College of Letters \& Science, University of Idaho, Moscow, ID 83843

ROYALTY, JAMES E., Language Media Center, University of Maryland, College Park, MD 20742

RUSDEN, SCV, Serials Librarian, H. B. Sarjeant Library, 662 North Blackburn Road, Clayton 3168, Vic., Australia

RUTGERS UNIVERSITY, Kilmer Area Library, New Brunswick, NJ 08903

RYAN, BRICE, Chairman, St. Vincent College, Latrobe, PA 15650

SAM HOUSTON STATE UNIVERSITY, Foreign Language Department, Huntsville, TX 77340

SAMPON, VICTOR, Director Language Lab, Wayland Academy, Beaver Dam, WI 53916

SANANVANIT, MS. KWANCHAI, Sea Enterprises Co., Ltd., Paris Theatre, 292/15-16 Luk Luang Road, Bangkok 3, Thailand

SANCHEZ-BERROA, ROGER A., Director, Wesleyan University, Language Lab, 116 Fisk Hall, West Station, Middletown, CT 06457

SANCHEZ, MARY, Supervisor Foreign Lang, Clearwater Public Schools, 1960 E. Druid Road, P.O. Box 4688, Clearwater, FL 33518 
SAYLOR, LESLIE G., Language Lab, Curtin B-31, 3225 Downer Ave., Milwaukee, WI 53201

SCARTH, MARGARET, Asst. Professor-Spanish, Erindale College, University of Toronto, 3359 Mississauga Road, Clarkston, Toronto, ON, Canada

SCHEWE JR., EDWARD, 419 South Taylor Avenue, Oak Park, IL 60302

SCHOBEL, DIANNE, 7801, Sagamore Drive, Cincinnati, OH 45236

SCHOOL FOR OVERSEAS STUDENTS, Library, Attn: Ms. Kidansky, Goldsmith Building, Mt. Scopus, Jerusalem, Israel

SCHRADER, ERNST, College Department, John Wiley \& Sons, 605 Third Avenue, New York, NY 10016

SCHULTZ, DAVID, Herbert $H$. Lehman College, City University of New York, A/V Language Department, Carment B-38, Bedford Park Blvd. West, Bronx, NY 10468

SCHWARZ, LUANNE, Language Laboratory, 402 Humanities Building, Emory University, Atlanta, GA 30322

SCOTT JR., JOSEPH R., Middlesex School, Concorn, MA 01742

SEAMEO REG. ENG. LAND., Librarian of Info Center, 30 Orange Grove Road, Singapore, 10, Singapore

SEIDEL, J., Language Laboratory, University of Victoria, P.O. Box 1700, Victoria, BC, Canada

SENG, MARK W., Assoc. Professor, University of Texas, 2505 Princeton Drive, Austin, TX 78741

SEYDOUX, ALINE, Associate Director, Learning Resource Center, Loyola University, 7101 W. 80th Street, Los Angeles, CA 90045

SHEEHAN, JOSEPH H., Director, Language Learning Technology, School of Lang. \& Ling., Georgetown University, Washington, DC 20057

SHIRLEY, CARL R., Director F. L. Resources Center, University of South Carolina, Columbia, SC 29208

SIMON FRASER UNIVERSITY, Library-Acquisition Div., Burnaby, BC 2, Canada

SLAVENS, MARJORIE, Chairperson, Foreign Language Dept., Rockford College, Rockford, IL 61101

iLESSINGER, LEAH, Coordinator, Post College, Learning Laboratory, Country Club Road, Waterbury, CT 06708

SMITH, PHILIP D., DR., Professor, Languages, West Chester State College, M 115, West Chester, PA 19380

SMITH, WILLIAM FLINT, Department of Mod. Lang., Purdue University, Lafayette, IN 47907

SMITHER, WILLIAM J., Director of Language Lab, Tulane University, New Orleans, LA 70118

SNOUSE, PAT, Instructional Assistant, Davidson College, Davidson, NC 28036

SOLDEVILLE, DR. F., Director, Scripps College, Language Lab, Balch Hall \#41, Claremont, CA 91711

SOMMER, URSULA, Asst. Dir. Language Lab, Fairleight Dickinson Univ., FlorhamMadison Campus, 285 Madison Avenue, Madison, NJ 07940 
SOUTHERN ILLINOIS UNIVERSITY, Morris Lib, Periodical Department, Carbondale, IL 62901

SOUTHWESTERN COLLEGE, Library, 900 Otay Lakes Road, Chula Vista, CA 92010

SOUTHWESTERN, UNIV. OF LOUISIANA, Serials Department, Dupre Library, 302 E St. Mary Blvd., Lafayette, LA 70504

SPINKS, O. R., Director, Humanities B 16, Suny At Albany, Albany, NY 12222

ST. ANDREWS, UNIVERSITY OF MODERN LANG. LIBRARY, Attn: Ms. Kohn, Buchanan Building, Union Street, St. Andrews, Fife KY16 9PH, Canada

ST. JOHN'S UNIVERSITY, Language Laboratory, Attn: Dr. Sallese, St. Albert's Hall Rm. 103, Grand Central \& Utopia, Jamaica, NY 11439

ST. MICHAEL'S COLLEGE, Attn: Norman Lacharite, Director-International Stud., Winooski, VT 05404

STADT-UND UNIVERSITATS-, 134-138, D Frankfurt 1, Germany

STANFORD UNIVERSITY, Library-Serials Department, Stanford, CA 94305

STEELE, KATHERINE L., DR., University of Florida, Office of Instructional Res, 450 Library East, Gainesville, FL 32611

STELLeNBOSCH, UNIV. OF, Library, Private Bag 5036, Stellenbosch, South Africa

STERN, RHODA, Supervisor Language Lab, Baruch College, City University of New York, 17 Lexington Avenue, PO Box 447, New York, NY 10010

STREI, PROFESSOR G., Head Language Lab Division, AV Department, H399 Concordia University, 1455 De Maisonneuve Blvd., Montreal, PQ H3G 1M8, Canada

STUART, MS. BOBBI CIRIZA, 6511 76th Street, Cabin John, MD 20731

SULAN, ZAINUDDIN, For Ketua Kajian, Pusat Bahasa, Institiut Teknoloji Mara, Shan Alam, Selangor, West Malaysia

SUMMIT IR. HIGH SCHOOL, Attn: Ms. Helen Landry, Summit, NJ 07901272 Morris Ave.

SUTTON, LOIS M., DR., Professor of rfench, Baylor University, Waco, TX 76703

SUZUKI, JUICHI, 610-205 Vematsu-Cho, Kishiwada-Shi Osaka 596, Japan

SWANSON, SEVERIN A., Language Lab Director, University of Wisconsin, Oshkosh, WI 54091

SWIFT, MS. M. H., Morgan Park Academy, 2153 West 111th Street, Chicago, IL 60643

SWINYARD, SHARON I., Professor/Director, St. Patrick's College, Language Lab, Box 151, Mountain View, CA 94042

SYDNEY, UNIVERSITY OF FISCHER LIBRARY, Serials Division 134881, Sydney, New South Wales 2006, Australia

TAKACX, LASZLO, Area Chairman, Administration Center, 5933 West 115th Street, Worth, IL 60482

TANDBERG, OF AMERICA INC.. President, Kjell S. Hoel, Labriola Court, Armonk, NY 10504

TAYLOR IR., WALTER C., Department of Spanish, Upsala College, East Orange, NJ 07019

TEACHERS COLLEGE LIBRARY, Serials Dept., 525 West 120th Street, New York, NY 10027

TENAGLIA, AMEDEO N., Rutgers University, Spanish Department, Camden, NJ 08682 
TENNESSEE, UNIVERSITY OF, Library Serials Division, Knoxville, TN 37916

TEXAS EDUCATION AGENCY, Reseource Center Library, 201 East Eleventh Street, Austin, TX 78701

TEXAS STATE UNIVERSITY, NORTH, Acquisitions Services, Ms. L. Evans, Box 5138 NT Station, Denton, TX 76203

TEXAS TECH UNIVERSIY, Germanic \& Slavic Languages, University Library, P.O. Box 4579, Lubbock, TX 79409

TEXAS-AUSTIN, UNIVERSITY OF, John G. Bordie, Director, Foreign Language Education, Center Library, Austin, TX 78712

TEXAS-AUSTIN, UNIVERSITY OF, Attn: Dr. Van Olphen, Director, Language Laboratory, Batts Hall 1, Austin, TX 78712

THIBAULT, CLAUDE, Notre Dame De Foy, Cap-Rouge, PQ GOA-IKO, Canada

THORNE, E. F., Associate Professor, West Liberty State College, West Liberty, WV 26074

TOKYO-SEITOKU-TANKI-DAIGAKU, Library (MZ), 7-13, Jujodai-1-Chome, Kita-Ku, Tokyo, Japan

TOLEDO, UNIVERSITY OF, Carlson Library, Serials Department, Toledo, OH 43606

TRACY, ROBERT K., Director Language Lab, Ridgewood High School, Ridgewood, NJ 07450

TUBINGEN, UNIVERSITY KASSE, Zentrum-Neue Lernverfahren, Federal Republic of Germany, Germany

UHLER, SUSAN D., 6 Park Overlook Ct., Carderock, MD 20034

UMMEL, CAROLYN M., Valley View High School, R. R. 2, Box 3370, Germantown, OH 45327

UNION COLLEGE, Library, 3800 S. 48th Street, Lincoln, NE 68506

UNIVERSITEITSBIBLIOTHEEK, 1439, A. 324. 639, Eras Muslaan 36, Nijmegen 1, Holland

UNIVERSITY LIBRARY, Serials Dept.-Kardex, Governors State University, Park Forest S., IL 60466

USAF ACADEMY, Academy Library, MK For F05611-73-M-A646, CO 80840

VALACHOVIC, JAROSLAV, 162 Westwood Road, Columbus, OH 43214

VALESKA, MARY ANN, COLORADO STATE UNIVERSITY, Department of English, Intensive English Program, Fort Collins, CO 80523

VAN WALK, GRACE R., Instructor, Grants Pass High School, 522 NE Olive, Grants Pass, OR 97526

VEITZ, SISTER M. FRANCES, Assistant Professor, Holy Family College, Grant \& Frankfort Avenue, Philadelphia, PA 19114

VELTMAN, PROF. HUGH E., Grand Valley State College, P.O. Box 58, Lamont, MI 49430

VERNICK, JUDY A., Director Language Lab, University of Pittsburgh, 732 C of L, Pittsburgh, PA 15260

VERRETTE, VICTOR S., Language Lab Director, Grinnell College, Grinnell, IA 50112

VIJAYAKUMAR, E. K., Thurber Comprehensive H. S., 15 Orilla Park, Red Deer, AB, Canada 
VIRGINIA, UNIVERSITY OF, Jackie Tanner, Director, Language Laboratroy, 221 Cabell Hall, Charlottesvl, VA 22903

VITI, ROBERT M., Language Laboratory Director, Gettysburg College, German Department, Gettysburg, PA 173255

VOGE, DR. WILFRIED, Language Laboratory, Room 263 Humanities Hall, University of California, Irvine, CA 92717

VON HUMBOL, TUR YLIOP ALEX, Kielilaboratorio, 20500 Turku 50, Finland

WARKENTIN, HENRY, DR., Foreign Language Department, Shippensburg State College, Shippensburg, PA 17257

WASHINGTON UNIVERSITY, Library, Acquisitions Department, St. Louis, MO 63130

WATSON, JOHN A., DR., Director Language Lab, Virginia Union University, Richmond, VA 23220

WAYNE COUNTY INTERMEDIATE, School District, Attn: Ms. B. Katz, Professional Resource Center, Information Services, 33500 Van Born Road, Wayne, MI 48184

WEATHERFORD, MS. HARRIET T, Language Lab Director, University of Illinois, G-70 Foreign Languages, Urbana, IL 61801

WEIBLE, DR. DAVID M., Director, Audio Info Service, University of Illinois At, Chicago Circle, Box 3438, Chicago, IL 60680

WEINKAUf, ARNOLD L., DR., Assoc. Prof. of Languages, Michigan Technological Univ., Houghton, MI 49931

WELLESLEY SR. HIGH SCHOOL, Foreign Language Department, 50 Rice Street, Wellesley HIs, MA 02181

WELLS, STEPHEN, Lab Director ELS Language Center, L666 Cross Center Drive, Norman, OK 73069

WEST CHESTER SJATE COLLEGE, Francis Green Library, West Chester State College, West Chester, PA 19380

WEST GEORGIA COLLECE, School of Arts \& Science, Department of Foreign Lang., Carrollion, GA 30117

WEST TEXAS STATE UNIVERSITY, Cornette Library, Box 748 W. T. Station, Canyon, TX 79016

WESTERN ONTARIO, UNIVERSIY OF, General Library, Periodicals Department, London, ON N6A 3K7, Canada

WIESE, PEER, Director Language Lab, Southern Connecticut College, 501 Crescent Street, New Haven, CT 06515

WILLECKE, F. H., DR., Chairman Language Dept., Wagner College, Staten Island, NY 10301

WILLIAMS, CHARLES B., Director Language Lab, State University of New York, Oswego, NY 13126

WILLIAMS, JAMES J., Director Language Lab, Elmhurst College, 190 Prospect Avenue, Elmhurst, IL 60126

WILLIS, IONE, R. R. 3, Milton, KY 40045

WILLIS, ROBERT M., Prolessur, Communications Arts \& Sciences, 829 Shimkin Hall, 50 W. 4th St., New York, NY 10003

WILLISTON, MRS. JACQUELINE, Director Language Lab, Connecticul College, Box 1524, New London, CT 06320 
WILTON SENIOR HIGH SCHOOL, Library, 395 Danbury Road, Wilton, CT 06897 WIPF, JOSEPH, 121 East Knox Drive, W. Lafayette, IN 47906

WISCONSIN, UNIVERSITY OF, Library, Serials Department, Milwaukee, WI 53201

WISCONSIN, UNIVERSITY OF, Serials Department-Library, 728 State Street, Madison, WI 53706

WISCONSIN, UNIVERSITY OF, Parkside, Library, Serials Division, Wood Road, Kenosha, WI 53140

WOLLSTEIN, JOHN, MR., Program Specialist-For Langs, Department of Education, 1270 Queen Emma Street, Room 1201, Honolulu, HI 96813

WYNN, WALTER W., 975 Ventura Avenue, Albany, CA 94707

YANNEY, DOUGLAS M., Director Language Lab, Wichita State University, Box 11, Wichita, KS 67208

YELLIN TEACHER'S SEMINARY, Beth-Hakerem, Jerusalem, Israel

YIN, MS. PHILLIPPA B., Director Language Lab, Cleveland State University, Cleveland, OH 44115

YORK UNIVERSITY, Scott Library, Central Serials Records, 4700 Keele Street, Downsview, ON M3) 2R2, Canada

ZARR, VALOIS A., Chairman German Department, East High School, 1134 South 17th Street East, Salt Lake Cy, UT 84108

ZAVATtero, Fred H., Professor, Hosei University, Building 62, Fujimi-Cho, ChiyodaKu, Tokyo, Japan

ZIMMERMAN, DOROTHY M., SISTER, Mount Mary College, Language Lab, Milwaukee, WI 53222

ZENICK, MANUEL C., U.S. Peace Corps, American Embassy, P.O. Box \#PC-15-761, APO, San Francisco, CA 96346

ZIMMERMAN, M., MRS., Language Lab, Amherst College, Amherst, MA 01002

ZINGIS, ANDRIS, Director Language Lab, University of Cincinnati, 372 Old Chemistry Bldg., Cincinnati, OH 45221

\section{The Many Faces william Price Fox of Humor in Twentieth-Century $\quad \begin{array}{ll}\text { Marry Golden } \\ \text { Literature }\end{array}$}

Registration Form Eighth Annual Conference

University of Louisville 28 and 29 February 1980

Name_Lept. $\_$Registration Fee $(\$ 20.00)$

Address

Checks are to be made to: University of Louisville. Mail the registration form to: Marilyn V. Schuler, Conference Chairperson, Department of Modern Languages, University of Louisville, Louisville, Kentucky 40208.
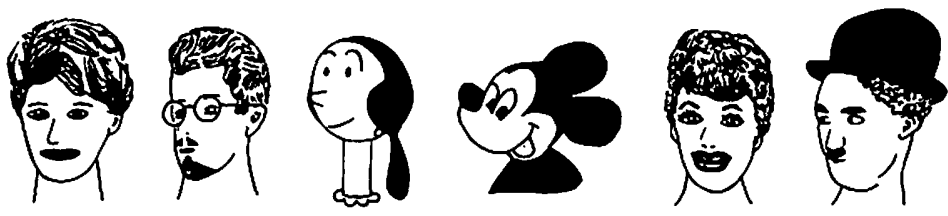